\title{
Iterative Optimization of an ESI IT Mass Spectrometer Using Regular Simplex and a Multivariate Target Function Representing the S/N Ratio
}

\author{
Elisa Robotti, Fabio Gosetti, Eleonora Mazzucco, Davide Zampieri, Emilio Marengo
}

Department of Environmental and Life Sciences, University of Eastern Piedmont, Viale Michel 11, 15121 Alessandria, Italy

\begin{abstract}
Standard automatic tuning for mass spectrometry usually exploits a one-variable-at-a-time approach. This method suffers from important drawbacks: the target function selected for optimization improves the signal of a single channel or a pool of channels without considering noise; the interactions between the parameters are not evaluated. The optimization of the experimental settings of an ESI IT mass spectrometer is carried out here by a multivariate procedure exploiting a target function representing the $S / N$ ratio calculated by principal component analysis and a regular simplex optimization algorithm. A preliminary feasibility study was performed since the target function must be sensitive to the changes in the experimental conditions applied during the iterative tuning and be free from drifts. The feasibility study was carried out to evaluate: the presence of memory effects; the size of the variations in the S/N ratio; the number of scans needed to generate a reliable $\mathrm{S} / \mathrm{N}$ ratio; the concentration of the multistandard mixture to use during tuning; the experimental duration required to achieve $\mathrm{S} / \mathrm{N}$ stability when the experimental settings are modified. The feasibility study led to the identification of the best protocol to accomplish the tuning, while simplex optimization allowed the $\mathrm{S} / \mathrm{N}$ ratio to be improved by about $70 \%$ with respect to the default conditions suggested by the manufacturer.
\end{abstract}

Key words: Mass spectrometry, Electrospray ionization, Simplex optimization, Signal-to-noise evaluation, Principal component analysis, Instrumental tuning

\section{Introduction}

$\mathrm{T}^{\mathrm{s}}$ he application of mass spectrometry (MS), whether coupled to liquid chromatography, gas chromatography or used by itself, requires the optimization of the various parameters of the mass spectrometer relating to both ion source and analyzer, with the aim of improving analytical sensitivity and efficiency. The optimization is usually accomplished by implementing automatic tuning procedures [1-4] that are directly managed by the software provided with the mass spectrometer, and it generally suffers from some important drawbacks. Standard automatic tuning is usually carried out by the OVAT (one variable at a time)

Correspondence to: Emilio Marengo; e-mail: marengoe@tin.it approach [5], based on the independent optimization of each parameter: for each experimental parameter, the setting that provides the largest increase in intensity in the corresponding mass spectrum is usually searched for.

The two most important drawbacks of standard automatic tuning are: (1) the target function selected to carry out the optimization, which usually consists of the measured signal (it should increase during the tuning); (2) the use of the OVAT approach, which does not take interactions into consideration, and often provides poor optimal settings.

The automatic tuning approach generally involves applying small changes to each experimental parameter to improve the signal of a single or a pool of selected $\mathrm{m} / \mathrm{z}$ values (channels); the channels can change according to the 
standard mixture suggested by the manufacturer or the choice of standard mixtures that are deemed suitable to cover the overall $\mathrm{m} / \mathrm{z}$ range [1-4]. In all cases, whichever individual channel or pool of $\mathrm{m} / \mathrm{z}$ values is selected, the tuning procedure provides the overall increase in intensity of the selected channels but does not consider the concurrent increase in noise. This first problem has already been addressed by our research group in a previous work [6], where a method for separating signal from noise in complex mass spectra was proposed. This method allows the automatic identification of the $\mathrm{S} / \mathrm{N}$ ratio from mass spectra across the entire $\mathrm{m} / \mathrm{z}$ range, and it is based on the use of principal component analysis (PCA).

However, the identification of a suitable target function that takes into account both signal and noise does not, by itself, permit the effective optimization of the experimental parameters; in fact, the use of OVAT optimization makes it impossible to evaluate the interactions between the parameters that are optimised. This problem often prevents the optimal settings from being identified, thus providing conditions that can be further improved.

Another issue relates to the number of parameters optimised by standard automatic tuning, which, in general, does not incorporate all of the parameters available; some of them are fixed by the manufacturer as it is assumed that they do not have a significant effect on the final target function. However, this is not always the case: parameters that are fixed by default may not have a significant effect when they are considered independent factors, but they may play a significant role in interactions with other factors.

One effective alternative is to use iterative optimization algorithms, like the simplex method [5, 7-11]. Such methods take into account the interactions between variables and identify the optimal conditions as a function of all factors considered. The main advantage of this is the possibility of identifying new experimental settings at each iteration, thus leading to an improvement in the target function. This feature makes these methods particularly interesting for automatic tuning: if tuning needs to be stopped, the procedure provides the best experimental conditions obtained at that moment, which are usually better experimental settings than the the starting ones. The simplex optimization procedure has already been widely applied both to mass spectrometry and other analytical procedures [7, 12-20].

A particularly effective approach to optimization could involve the use of the simplex algorithm coupled to the target function based on the multivariate definition of the $\mathrm{S} / \mathrm{N}$ ratio [6].

With this in mind, the present work targets the optimization of the experimental settings of an ESI (electrospray ionization) IT (ion trap) mass spectrometer [21-24], which is not coupled to separation techniques, by regular simplex. The optimization phase is preceded by a feasibility study of multivariate tuning: this preliminary study is necessary since the chosen target function must be sensitive to the changes in the experimental conditions applied during iterative tuning and be free from natural drifts and the oscillation of the experimental system, which may hide the desired improvements. Too often, complex instrumentation that provides complex experimental responses, such as a mass spectrometer, is used without user control and feedback, while potential criticalities must be carefully evaluated in order to achieve reliable optimization.

Therefore, the feasibility study is carried out in order to:

1. Evaluate the experimental error in the target functiondefined as the $\mathrm{S} / \mathrm{N}$ ratio calculated as in [6]

2. Check whether variations that are intentionally applied to the operating parameters (during optimization) produce statistically relevant variations (larger than experimental error) in the target function

3. Verify the presence of memory effects (i.e. whether the order in which the experiments are carried out has an effect)

4. Identify the optimal concentration of the multi-standard mixture to use in the tuning procedure

5. Identify the optimal number of scans to record in order to generate a reliable $\mathrm{S} / \mathrm{N}$ ratio

All of these aspects are evaluated below in order to produce a workflow that can be followed each time a complex optimization study must be performed. The final optimal settings identified by simplex are compared to the default settings suggested by the manufacturer.

\section{Theory}

\section{Simplex Optimization}

Simplex optimization $[5,7-11]$ is an iterative procedure that allows the simultaneous optimization of $p$ parameters. An initial simplex-represented by a set of $p+1$ initial experiments that sample the experimental domain-must be selected. In the case described in this work, the first experiment of the initial simplex consisted of the default conditions suggested by the manufacturer. The other $p$ experiments of the simplex were generated by applying single variations to each parameter independently, according to the procedure described by Spendley et al. [11]: in this way, the initial simplex homogeneously explores the overall experimental domain in all directions.

Once the experiments of the initial simplex have been performed, they are evaluated according to the target function selected, and an iterative procedure begins; during each iteration, the worst experiment is identified and projected with respect to the centroid of the others. If the new experiment provides a better result, the worst experiment is replaced in the simplex by the new one; otherwise the second worst is projected. The procedure reaches convergence when all of the experiments of the simplex have been projected without identifying a new best experimental condition.

It is important to point out that simplex, being an iterative procedure, does not guarantee the identification of the global 
optimum - the procedure may converge to local optimal conditions. It is, however, a suitable approach to use in the present case, as it can be interrupted before convergence but still pinpoint new settings that represent an improvement on the initial ones.

\section{Experimental}

\section{Apparatus}

MS experiments were conducted by means of a Thermoquest LCQ Duo ion trap mass spectrometer from Finnigan (San Jose, CA, USA), which was equipped with ESI. The MS software used was Xcalibur 2.0 SR2 (ThermoElectron Corporation, San Jose, CA, USA). Statistical calculations were performed and graphical representations obtained using Excel 2003 (Microsoft Corporation, Redmond, WA, USA), The Unscrambler v.9.2 (Camo, Oslo, Norway) and Statistica v.7.1 (StatSoft Inc., Tulsa, OK, USA). Matlab (R2007b; The Mathworks, Natick, MA, USA) was used to develop homemade algorithms for the automatic management of the data collected by the instrument and for the automatic calculation of the $\mathrm{S} / \mathrm{N}$ ratio from mass spectra.

\section{Preparation of the Calibration Solution}

The calibration and tuning solution adopted was the one suggested by the manufacturer, which consisted of the use of a complex standard mixture of caffeine, MRFA (a tetrapeptide: methionine-arginine-phenylalanine-alanine) and Ultramark 1621. This solution is characterised by a pattern of $\mathrm{m} / \mathrm{z}$ values that cover the entire $\mathrm{m} / \mathrm{z}$ range available to the MS analyzer $(\mathrm{m} / \mathrm{z} 50-2000)$. The standard mixture is characterised by high concentration and long persistence [26, 27].

The solution of caffeine, MRFA, and Ultramark 1621 was prepared in a mixture solvent (acetonitrile, methanol, water) containing $1 \%$ of acetic acid. A calibration solution of $5.0 \mathrm{~mL}$ was prepared by mixing $100.0 \mu \mathrm{L}$ of caffeine methanol solution $\left(1 \mathrm{mg} \mathrm{mL}^{-1}\right), 5.0 \mu \mathrm{L}$ of MRFA methanol/ water $50 / 50 \mathrm{vol} / \mathrm{vol}$ solution $\left(5 \mathrm{nmol} \mu \mathrm{L}^{-1}\right)$, and $2500 \mathrm{~mL}$ of Ultramark 1621 acetonitrile solution $(0.1 \% \mathrm{vol} / \mathrm{vol})$ with $50.0 \mu \mathrm{L}$ of glacial acetic acid and $2.340 \mathrm{~mL}$ of $50 / 50$ methanol/water solution. The calibration solution was preserved in the dark in the refrigerator at $4{ }^{\circ} \mathrm{C}$, and remained stable for about 2 months.

\section{Reagents}

Ultrapure water was produced by a Millipore Milli-Q system (Milford, MA, USA). Methanol CHROMASOLV (>99.9\%) and caffeine $(99 \%)$ were acquired from Sigma-Aldrich (Milwaukee, WI, USA). HPLC-grade acetonitrile was acquired from Merck (Darmstadt, Germany).

The MRFA peptide MS standard was purchased from Research Plus (Manasquan, NJ, USA), and the Ultramark 1621 MS standard from Lancaster Synthesis (Pelham, NH,
USA). Glacial acetic acid for LC-MS was acquired from Fluka (Buchs, Switzerland).

\section{Mass Spectrometry Conditions}

High-purity nitrogen was used as nebulizer and helium $(>99.999 \%)$ served as the quenching agent. The AGC value was set automatically. Full-scan data acquisition was performed in positive ion mode over the range $\mathrm{m} / \mathrm{z} 50$ 2000. The maximum ionization time was set at $200 \mathrm{~ms}$ for 1 scan. ESI mass spectral profiles were obtained by direct

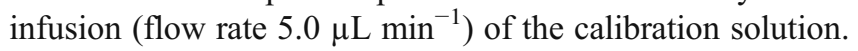
All dilutions required were derived from the solvent used for calibration.

\section{Results and Discussion}

\section{Evaluation of S/N from Complex Mass Spectra}

As described in a previous work [6], the $\mathrm{S} / \mathrm{N}$ ratio in ESI IT mass spectrometry can be effectively calculated via the principal component analysis (PCA) [10, 25] of complex mass spectra recorded over time. Applying PCA to a set of scans of a mass spectrum recorded across the full spectral range available, without scaling or centring the data, provides the first principal component (PC), which accounts for the average signal. It has been demonstrated that the residuals in this case account for the experimental noise. The intensity recorded in each channel is therefore separated into two contributions: one due to the signal (accounted for by the first PC), and one due to the noise (accounted for by the residuals). Figure 1 reports an example of the calculation of the $\mathrm{S} / \mathrm{N}$ ratio in an experiment carried out with the default settings suggested by the manufacturer. PCA was carried out on a $500 \times 1950$ data matrix (500 being the number of scans and 1950 the number of channels recorded) without any data pre-treatment. The first PC explains about 96\% (loadings are shown in Figure 1a) and represents, as expected, the average spectrum; the signal is represented by the scores of the first PC (Figure 1b), while the other PCs contain the noise (Figure 1c). Their ratio corresponds to the $\mathrm{S} / \mathrm{N}$ ratio that is calculated for each scan (Figure 1d).

This procedure is applied below to evaluate an average $\mathrm{S} /$ $\mathrm{N}$ ratio for each independent experiment.

\section{Feasibility Study}

The feasibility study is structured into a three-step procedure in order to address all of the main concerns pointed out in the "Introduction": (i) the experimental domain is identified together with the variations that will be applied to each parameter during the final simplex optimization; (ii) experiments are designed and carried out to evaluate experimental error and memory effects; (iii) the best operating conditions to carry out the final optimization by regular simplex are identified. 


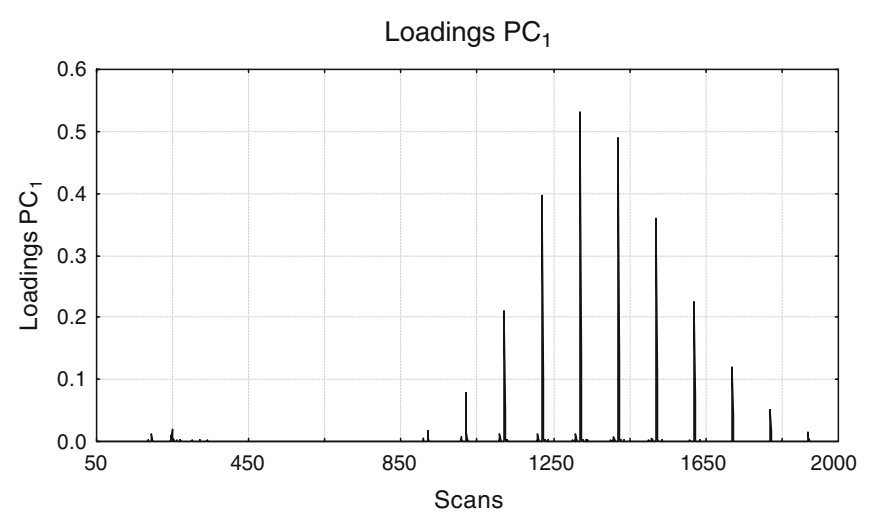

(a)

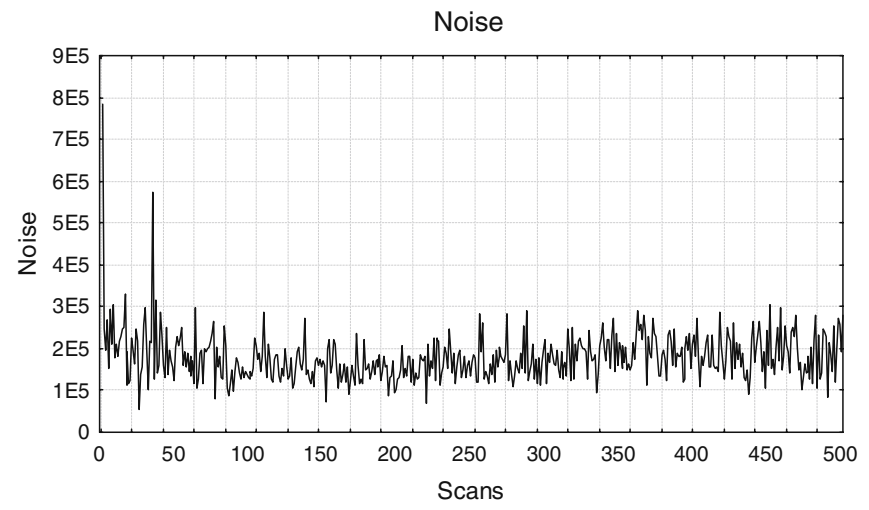

(c)

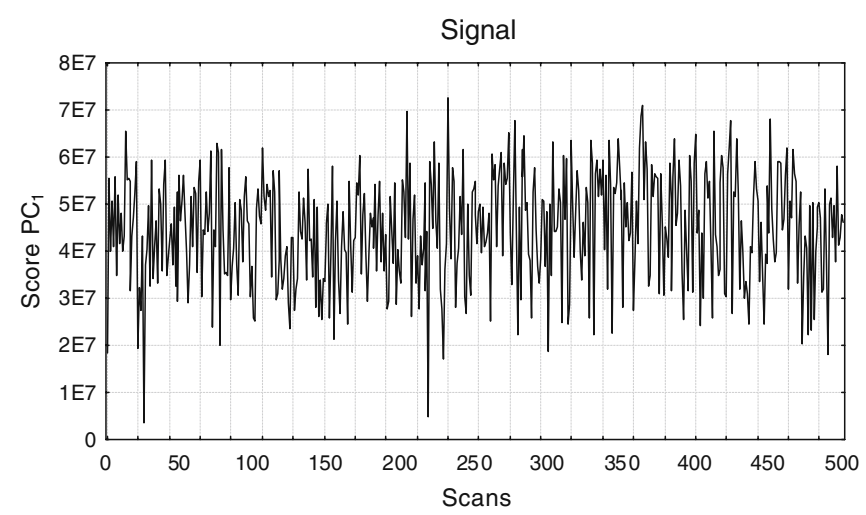

(b)

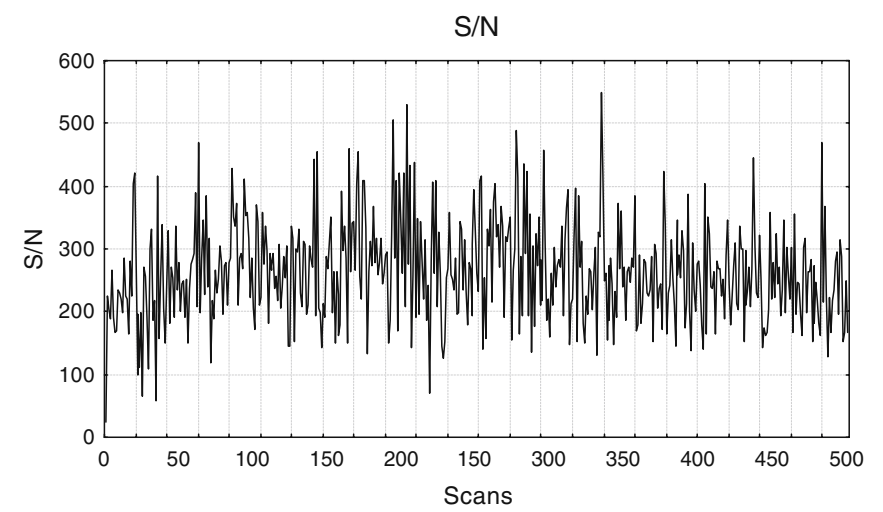

(d)

Figure 1. Example of the calculation performed for each experiment. The results refer to an experiment carried out with the default settings, recording 500 scans across the full $\mathrm{m} / \mathrm{z}$ range available. (a) The loadings of the first PC: loadings are reported on the $y$-axis and the number of scans on the $x$-axis. (b) The signal represented by the scores ( $y$-axis) of each scan ( $x$-axis) in the first PC. (c) The noise, represented by the standard deviation of the residuals of the first PC ( $y$-axis) for each scan ( $x$-axis). (d) The $S / N$ values $(y$-axis) for each scan $(x$-axis)

\section{The experimental domain}

Ten parameters were studied (Table 1), including those relating to the ionization source (sheath gas, spray voltage, capillary temperature and capillary voltage) and the ion trap MS analyser (tube lens offset, multipole offset 1 and 2, lens voltage, multipole RF and trap offset). For each parameter, Table 1 reports: (i) the label used for it in graphics and tables; (ii) the corresponding experimental domain available, described in terms of minimum and maximum values and the range of variation (difference between maximum and minimum); (iii) the variation applied to each parameter $(\Delta)$ in order to set up the initial simplex according to [11], and the sign of this variation (+ indicates variations towards the maximum value, while - indicates variations towards the minimum value).

\section{The experimental sequence}

Three experiments were selected to evaluate experimental error and memory effects: they represented the three most different experimental settings identified by the simplex procedure (Table 1, last three columns): experiment D corresponds to the default settings recommended by the manufacturer; $\mathrm{H}$ and $\mathrm{L}$ correspond to the application of all positive $(\mathrm{H}$, high) or all negative (L, low) variations to the default conditions. The variations applied correspond to $10 \%$ of the experimental domain (Table 1).

Experiments $\mathrm{H}, \mathrm{L}$ and $\mathrm{D}$ were arranged in a sequence (Figure 2a) of 14 experiments. After a stabilization period of about 5 minutes at the instrumental settings defined by experiment L, a given number of scans of the calibration standard solution over the $\mathrm{m} / \mathrm{z}$ range 50-2000 were recorded, providing the experimental results for experiment $\mathrm{L}_{\text {stable } 1}$ (Figure 2a). The experimental settings were then changed to those defined by D (i.e. increasing the values of all of the parameters simultaneously), and the same number of scans was recorded over the $m / z$ range $50-2000$ : this set provided the results for experiment $D_{\text {up } 1}$ (Figure 2a). After that, the experimental settings were changed to those corresponding to $\mathrm{H}$ conditions (i.e. increasing all of the parameters with respect to the settings for D), and another set of scans was recorded across the same $\mathrm{m} / \mathrm{z}$ range, providing 
Table 1. Parameters studied during optimization: experimental domain, expressed as minimum (min) and maximum (max) values available; range (expressed as max - min); variation applied during simplex $(\Delta)$; sign of the variation applied ( + indicates a movement of the initial simplex towards larger or more positive values, and - indicates a movement of the simplex towards smaller or more negative values). The last three columns report the values of each parameter for the experiment carried out under the default conditions (D), and for the two experiments representing all positive (H) or negative (L) variations of each parameter

\begin{tabular}{|c|c|c|c|c|c|c|c|c|c|}
\hline Parameter & Label & Min & Max & Range of variation & $\Delta$ & Sign of the variation & D (default) & $\mathrm{H}($ all +) & $\mathrm{L}($ all -) \\
\hline $\begin{array}{l}\text { Sheath gas } \\
\text { (arbitrary units) }\end{array}$ & ShGas & 20 & 80 & 60 & 10 & + & 20 & 26 & 20 \\
\hline Spray voltage $(\mathrm{kV})$ & SprayVolt & 2.00 & 6.50 & 4.50 & 0.75 & - & 5.00 & 5.45 & 4.55 \\
\hline Capillary temp $\left({ }^{\circ} \mathrm{C}\right)$ & CapTemp & 100.00 & 300.00 & 200.00 & 30.00 & + & 200.00 & 220.00 & 180.00 \\
\hline Capillary voltage V & CapVolt & 1.00 & 132.00 & 131.00 & 20.00 & + & 21.00 & 34.10 & 7.90 \\
\hline Tube lens offset (V) & TLens & 0.00 & 120.00 & 120.00 & 20.00 & + & 0.00 & 12.00 & 0.00 \\
\hline Multipole 1 offset (V) & Mult1 & -120.00 & -1.00 & 119.00 & 20.00 & - & -2.25 & -1.00 & -14.15 \\
\hline Lens voltage (V) & LensVolt & -132.00 & -3.00 & 129.00 & 20.00 & - & -30.00 & -17.10 & -42.90 \\
\hline Multipole 2 offset (V) & Mult2 & -132.00 & -2.00 & 130.00 & 20.00 & - & -7.50 & -2.00 & -20.50 \\
\hline $\begin{array}{l}\text { Multipole RF } \\
\text { (V peak to peak) }\end{array}$ & MultRF & 200.00 & 600.00 & 400.00 & 60.00 & + & 400.00 & 440.00 & 360.00 \\
\hline Trap offset DC (V) & TrapDC & -100.000 & -4.000 & 96.000 & 15.000 & - & -10.000 & -4.000 & -19.600 \\
\hline
\end{tabular}

the results for experiment $\mathrm{H}_{\text {up1 }}$ (Figure 2a). The other experiments of the sequence were performed in a similar way, according to the sequence indicated in Figure 2a. Looking at this figure, the overall sequence can be split into two replicated "increasing sequences", in which the direction of change of the parameters is towards larger or more positive values, and two replicated "decreasing sequences", in which the direction of change of the parameters is towards smaller or more negative values. A "final sequence" consisting of two D replications in a stationary condition (i.e. after stabilizing the settings for 5 $\min$ at the default conditions) is also present.

Each experiment is characterised by a subscript index, where "up" corresponds to a measurement taken during an increasing sequence, "down" to a measurement taken during a decreasing sequence, and "stable" to a stationary phase, where the previous experiment was carried out under the same experimental settings. The overall sequence is designed so that each experiment is replicated twice for each "up", "down" or "stable" condition.

The overall sequence was repeated while changing the number of scans used to evaluate the $\mathrm{S} / \mathrm{N}$ ratio for each experiment $(3,5,10,50,100,500$ scans were recorded $)$ at different concentration levels of the calibration standard mixture (the one suggested by the manufacturer, as well as dilutions of it at 1:10, 1:100 and 1:1000). The concentration level was varied since a previous study [6] pointed out the existence of saturation effects, which were probably due to the high concentration and persistence of the multi-standard. The results obtained from each experimental sequence were evaluated according to Figure 2b: replications of the same experiment were used to evaluate the experimental error (step 1 in Figure 2b). A comparison of replications of the same experimental setting $(\mathrm{H}, \mathrm{L}$ or $\mathrm{D})$ along increasing or decreasing sequences was made in order to evaluate the presence of memory effects (step 2 in Figure 2b); memory effects were not present if these replications were not statistically different one from each other. If this was the case, the H, L and D experiments could be compared (step 3 in Figure $2 b$ ). If $\mathrm{H}, \mathrm{L}$ and $\mathrm{D}$ provided statistically different results, the experimental conditions selected (number of scans, concentration of the standard, variations applied to the parameters, etc.) could be adopted in order to carry out an effective optimization.

Results will be briefly presented for the concentrated standard mixture, while an exhaustive discussion will be devoted to the dilution 1:100, which provided the best results.

\section{Evaluation of experimental error and memory effects}

The results for the concentrated standard solution are shown in Table $2 \mathrm{a}$ for 50, 100 and 500 scans. The table reports the average $\mathrm{S} / \mathrm{N}$ calculated for each experiment $(\bar{x})$ and the extremes of the corresponding confidence interval (C.I. min-max) at a confidence level of $95 \%$. The overall sequence of 14 experiments recorded with a given number of scans was separated into subsequences that were grouped two by two in order to compare the two increasing and the two decreasing sequences independently, thus allowing their reproducibility to be verified. A cross-comparison of increasing and decreasing sequences was used instead to verify whether the $\mathrm{D}, \mathrm{H}$ and $\mathrm{L}$ experiments provided similar $\mathrm{S} / \mathrm{N}$ ratios when performed in a different order.

Regarding the concentrated standard solution, no similar trends (from $\mathrm{L}$ to $\mathrm{H}$ for increasing sequences and from $\mathrm{H}$ to $\mathrm{L}$ for the decreasing ones) were detected for the number of scans recorded. The experimental error was too large (confidence intervals overlap) for 50 and 100 scans. For 500 scans, the error was much smaller due to the large number of scans recorded but the sequences were not reproducible: the two increasing (from $\mathrm{L}$ to $\mathrm{H}$ ) and the two decreasing (from $\mathrm{H}$ to $\mathrm{L}$ ) sequences showed quite different trends. 


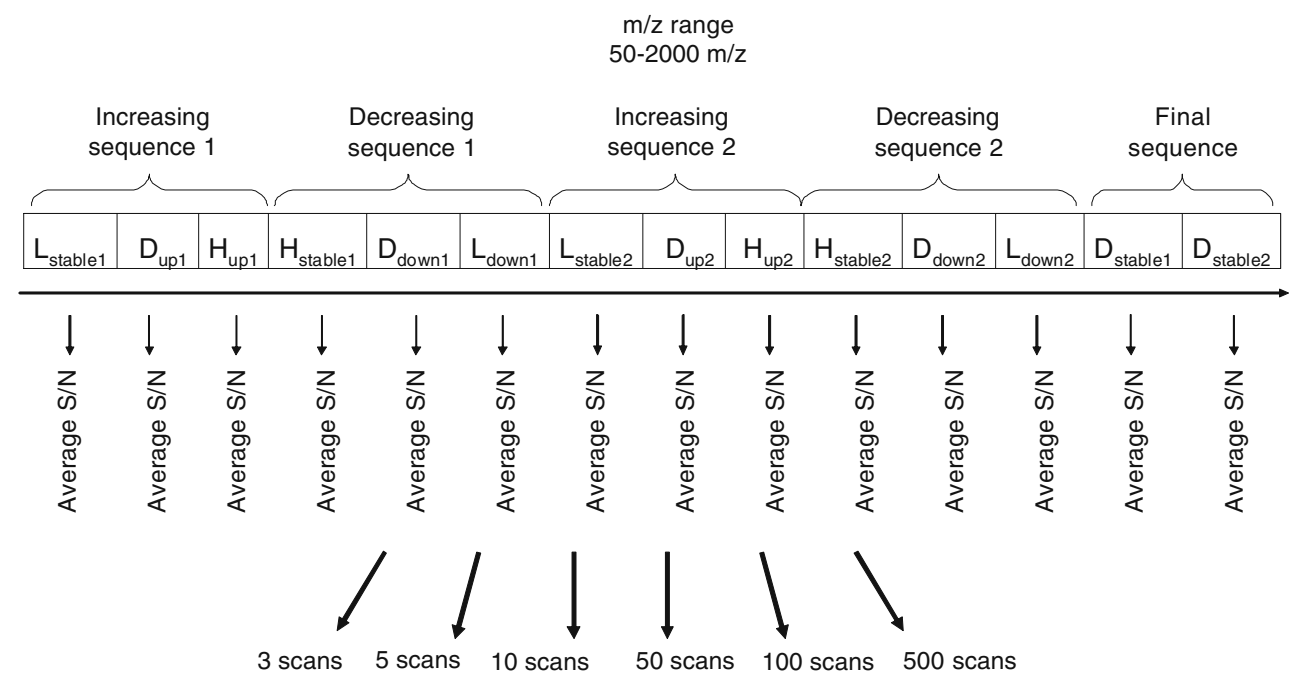

(a)

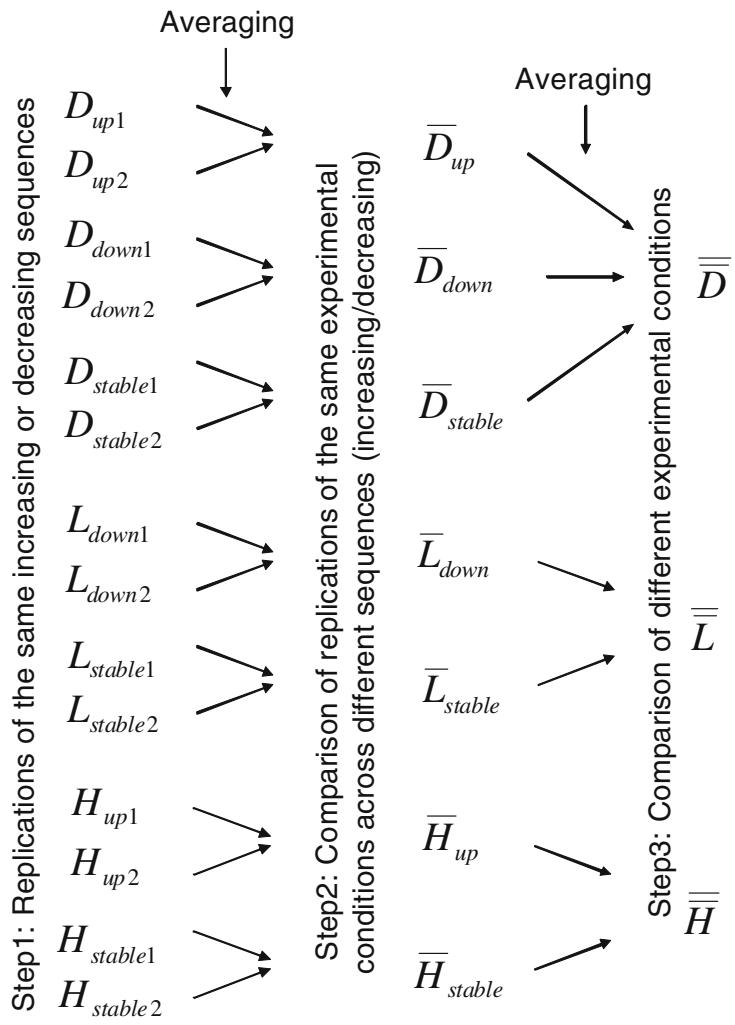

(b)

Figure 2. (a) Sequence of experiments carried out: $H, L$ and $D$ indicate the experimental conditions applied (Table 1); subscripts indicate the direction of change of the particular sequence. (b) Workflow illustrating how the results obtained from each sequence of experiments were analysed

No systematic behaviour was detected: $\mathrm{S} / \mathrm{N}$ values suffered from a general instability and lack of reproducibility, while no systematic memory effects were present. Obviously, carrying out any optimization under these conditions would lead to completely random final experimental settings!
The results for the standard mixture diluted 1:100 are reported in Table $2 \mathrm{~b}$ for 50,100 and 500 scans. While 50 scans gave less reproducible results and a larger experimental error (larger confidence intervals), 100 and 500 scans provided more reliable evaluations of the average $\mathrm{S} / \mathrm{N}$ values for each experiment, thus showing that the replicated 


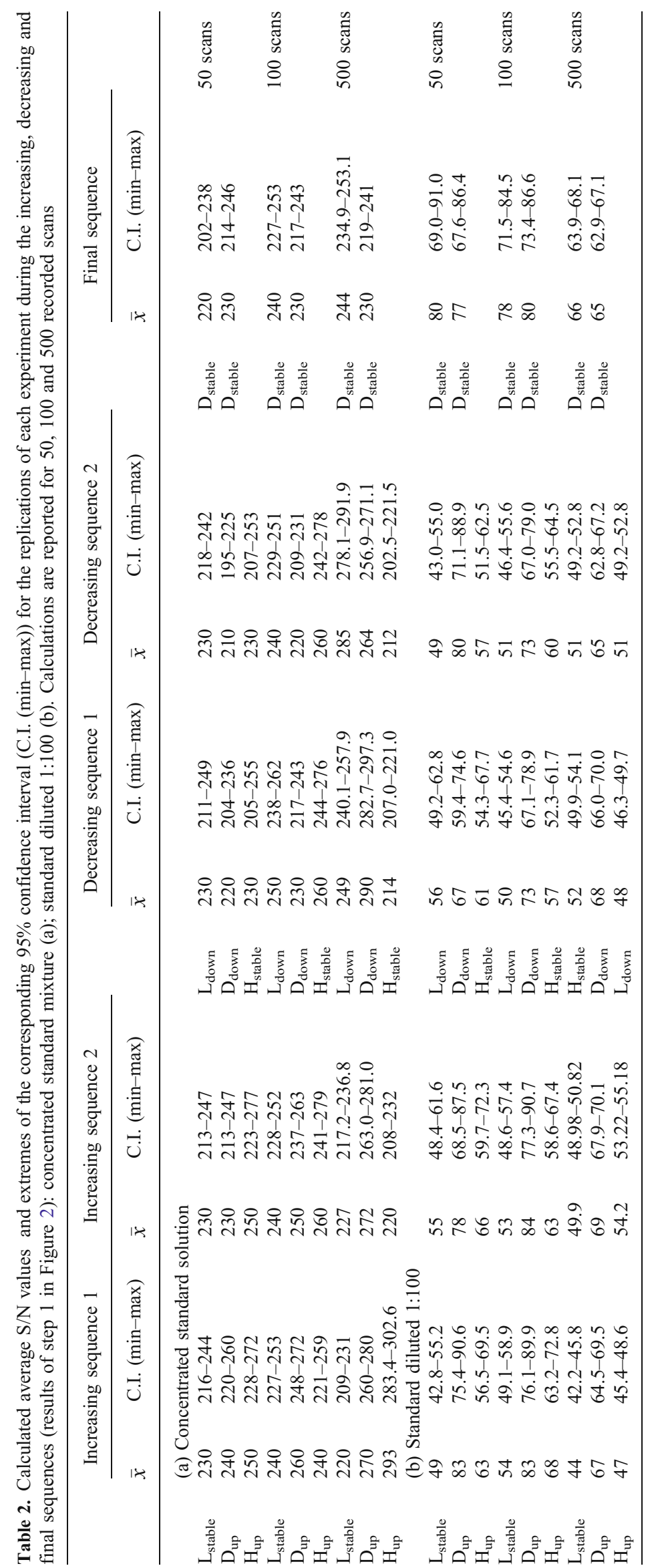




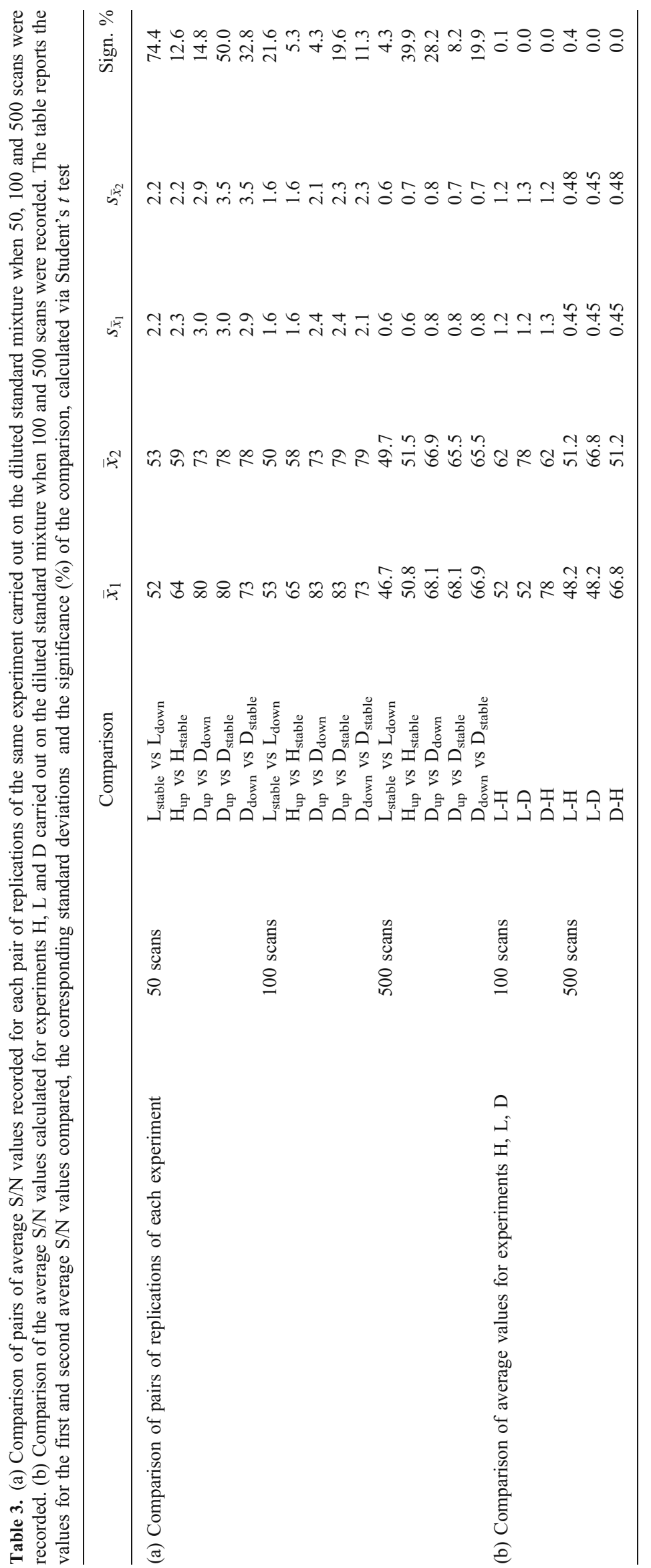


Table 4. Experiments of the initial simplex together with the calculated average $\mathrm{S} / \mathrm{N}$ ratio and its standard deviation. The last row of the table represents the experimental settings and the experimental response calculated for the best experiment obtained by the simplex optimization procedure

\begin{tabular}{|c|c|c|c|c|c|c|c|c|c|c|c|c|}
\hline Exp. & ShGas & SprayVolt & CapTemp & CapVolt & TLens & Mult1 & LensVolt & Mult2 & MultRF & TrapDC & $\bar{x}$ & $S_{\bar{x}}$ \\
\hline $\mathrm{S} 1$ & 20 & 5.00 & 200.00 & 21.00 & 0.00 & -2.25 & -30.00 & -7.50 & 400.00 & -10.000 & 165 & 1.5 \\
\hline $\mathrm{S} 2$ & 29 & 4.88 & 204.91 & 24.28 & 3.28 & -5.53 & -33.28 & -10.78 & 409.83 & -12.460 & 182 & 1.4 \\
\hline S3 & 22 & 4.35 & 204.91 & 24.28 & 3.28 & -5.53 & -33.28 & -10.78 & 409.83 & -12.460 & 192 & 1.7 \\
\hline $\mathrm{S} 4$ & 22 & 4.88 & 226.13 & 24.28 & 3.28 & -5.53 & -33.28 & -10.78 & 409.83 & -12.460 & 202 & 1.8 \\
\hline S5 & 22 & 4.88 & 204.91 & 38.42 & 3.28 & -5.53 & -33.28 & -10.78 & 409.83 & -12.460 & 193 & 1.6 \\
\hline S6 & 22 & 4.88 & 204.91 & 24.28 & 17.42 & -5.53 & -33.28 & -10.78 & 409.83 & -12.460 & 205 & 1.6 \\
\hline S7 & 22 & 4.88 & 204.91 & 24.28 & 3.28 & -19.67 & -33.28 & -10.78 & 409.83 & -12.460 & 1.0 & 0.28 \\
\hline S8 & 22 & 4.88 & 204.91 & 24.28 & 3.28 & -5.53 & -47.42 & -10.78 & 409.83 & -12.460 & 191 & 1.6 \\
\hline S9 & 22 & 4.88 & 204.91 & 24.28 & 3.28 & -5.53 & -33.28 & -24.92 & 409.83 & -12.460 & 186 & 1.7 \\
\hline $\mathrm{S} 10$ & 22 & 4.88 & 204.91 & 24.28 & 3.28 & -5.53 & -47.42 & -10.78 & 409.83 & -12.460 & 191 & 1.6 \\
\hline S9 & 22 & 4.88 & 204.91 & 24.28 & 3.28 & -5.53 & -33.28 & -24.92 & 409.83 & -12.460 & 186 & 1.7 \\
\hline $\mathrm{S} 10$ & 22 & 4.88 & 204.91 & 24.28 & 3.28 & -5.53 & -33.28 & -10.78 & 452.26 & -12.460 & 175 & 1.5 \\
\hline $\mathrm{S} 11$ & 22 & 4.88 & 204.91 & 24.28 & 3.28 & -5.53 & -33.28 & -10.78 & 409.83 & -23.060 & 114.0 & 0.86 \\
\hline S60 & 21 & 4.95 & 211.25 & 21.65 & 13.23 & -4.97 & -30.14 & -8.81 & 403.65 & -11.050 & 282 & 3.1 \\
\hline
\end{tabular}

increasing and decreasing sequences are very reproducible, above all in the case of 100 scans. Regarding the crosscomparison between increasing and decreasing sequences with 100 scans recorded, the decreasing sequences seemed to be shifted towards smaller $\mathrm{S} / \mathrm{N}$ values with respect to the increasing sequences: $D_{\text {up }}$ seemed larger than $D_{\text {down }}$, and the same happened for $\mathrm{H}_{\text {up }}$ and $\mathrm{H}_{\text {stable. This was not the case }}$ when 500 scans were recorded; here, the pairs of increasing and decreasing sequences gave similar values for the same type of experiment.

These results were confirmed by Student $t$ tests. Table 3a reports the results obtained when comparing, independently for 50, 100 and 500 scans, the increasing and decreasing sequences for the $\mathrm{B}, \mathrm{A}$ and $\mathrm{D}$ experiments. For each comparison carried out, $\bar{x}_{1}$ and $\bar{x}_{2}$ are the average $\mathrm{S} / \mathrm{N}$ values calculated for the first and second terms of the comparison, respectively, while $s_{\bar{x}_{1}}$ and $s_{\bar{x}_{2}}$ are their corresponding standard deviations. The last column in Table 3 reports the significance level calculated for each comparison: comparisons leading to a significance level smaller than $5 \%$ indicate that the two values compared are statistically different from one another.

As expected, 50 scans always gave significance levels of $>5 \%$, due to the high variability in the results. When 100 scans were performed, the two $\mathrm{H}$ experiments gave significance levels that were slightly larger than $5 \%$; the same happened for the two $D$ experiments $\left(D_{\text {up }}\right.$ and $\left.D_{\text {down }}\right)$. However, when 500 scans were performed, only the two B experiments gave significance levels of $<5 \%$; all the other experiments appeared to be reproducible across increasing and decreasing sequences.

Finally, the H, L and D experiments were compared in their entirety, by averaging all the average $\mathrm{S} / \mathrm{N}$ values obtained for the replicated increasing and decreasing sequences. The results of the Student $t$ tests (Table $3 \mathrm{~b}$ ) show that all of the experiments gave statistically different $\mathrm{S} / \mathrm{N}$ average ratios (all significance levels are much smaller than $5 \%$ ).
The sequence recorded with 500 scans provided the best results since it was characterised by the smallest variability (due to the larger number of replicated scans) and was free from memory effects.

\section{Identifying the best conditions to perform the optimization}

The best experimental procedure to carry out the subsequent optimization through regular simplex was therefore identified:

- The target function used during optimization was the $\mathrm{S} / \mathrm{N}$ ratio, calculated as already described [6]. For each experiment performed, the final target function is the $\mathrm{S} / \mathrm{N}$ averaged over all of the scans recorded.

- The concentration of the standard mixture: the standard mixture was diluted 1:100. This change in concentration level with respect to that suggested by the manufacturer is necessary to avoid memory and saturation effects; the experimental response is unstable and unreliable when a standard that is too concentrated is adopted. A dilution of 1:10 was also found to be too concentrated to completely avoid saturation effects, while a dilution of 1:1000 required a relatively large number of scans to provide reliable $\mathrm{S} / \mathrm{N}$ values.

- Number of scans for each experiment: a series of 500 subsequent scans was recorded for each experiment during optimization; using this number of scans avoided memory effects and reduced the variability of the final response.

\section{Optimization Through Regular Simplex}

The optimization of the ten parameters reported in Table 1 was then carried out by regular simplex. Table 4 reports the experiments of the initial simplex (S1-S11) as defined in [11], together with the calculated average $\mathrm{S} / \mathrm{N}$ ratio and its standard deviation. For each experiment, 500 full-range 


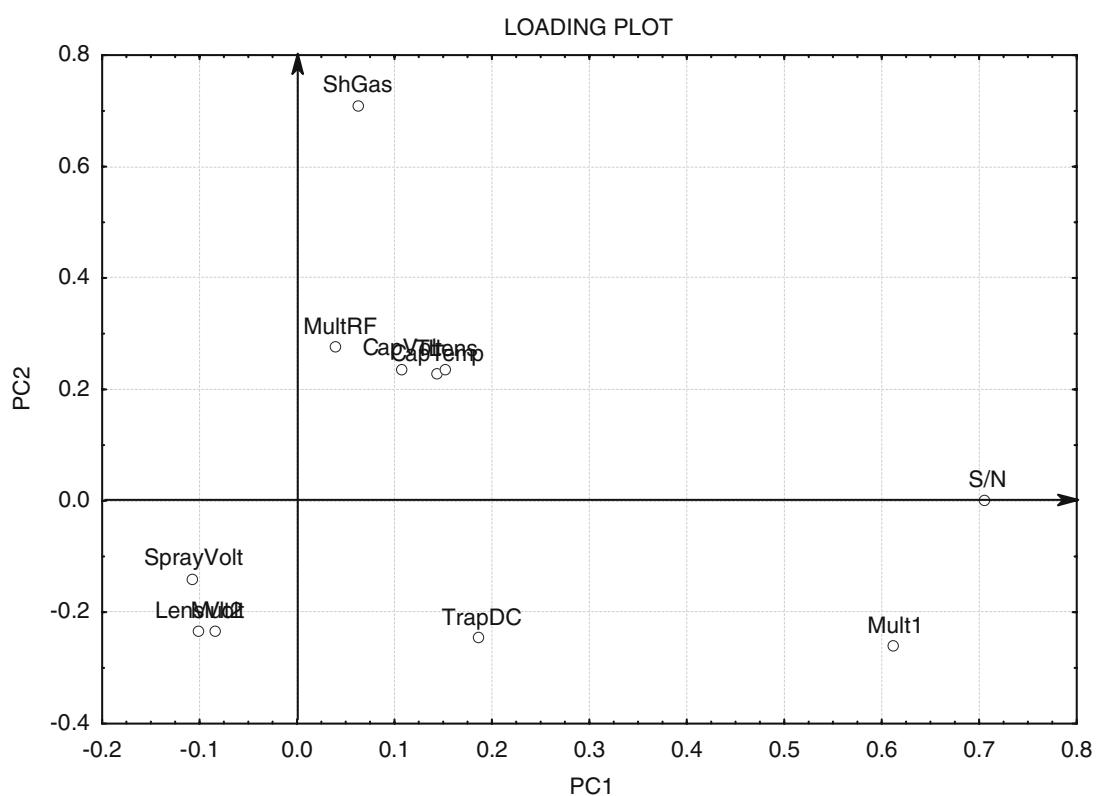

(a)

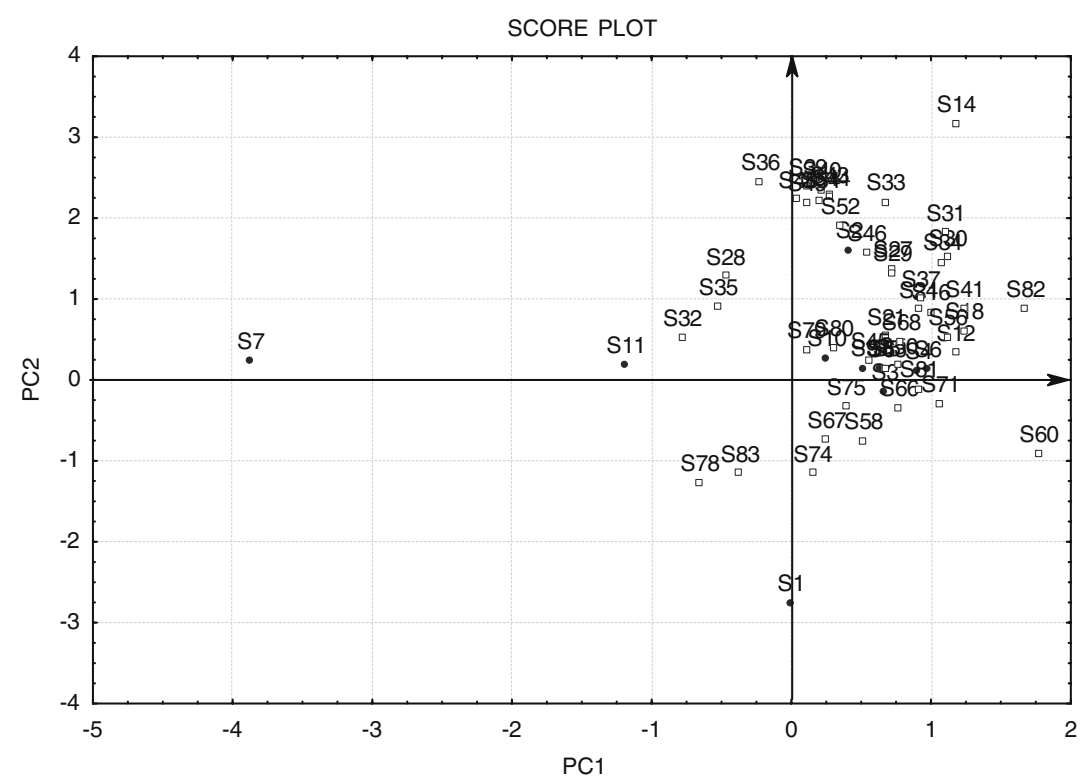

(b)

Figure 3. Results of the PCA applied to the initial simplex (further optimization experiments are projected into the space given by the first two PCs): loading plot (a) and score plot (b) of the first two PCs (experiments from the initial simplex are indicated as full circles, while subsequent optimization experiments are marked as empty squares)

mass spectra were recorded, and the calculated $\mathrm{S} / \mathrm{N}$ was averaged across all of the scans recorded.

The simplex procedure was stopped after 83 iterations.

Figure 3 reports the results of PCA carried out on the initial simplex, after autoscaling: the experiments of the initial simplex were described by the ten parameters that underwent optimization and by the experimental response S/ $\mathrm{N}$. PCA was used in this case as a visualisation tool to sum the results obtained from simplex optimization. The amount of variance explained by each PC shows that the data are quite uncorrelated: the first two PCs explain about $18 \%$ and
$10 \%$ of the overall variance, respectively. Looking at the loading plot (Figure 3a), the first PC mainly accounts for the $\mathrm{S} / \mathrm{N}$ value, which shows a large positive weight and seems to be mostly related to the value of multipole offset 1 (Mult1). The value of Mult1 corresponds to a direct current voltage applied to a multipole rod assembly. This value helps to define the translational kinetic energy of the ions within the assembly. More negative values of Mult1 probably result in more effective transfer across the assembly. If the values are too negative, however, they can cause multipole region ion source collision-induced dissociation. The second PC mainly 
accounts for the sheath gas (ShGas) at positive weights, which is related to the production of a fine mist during sample nebulisation. Since this parameter is mainly accounted for by the second PC, it appears to be uncorrelated with the calculated $\mathrm{S} / \mathrm{N}$ ratio.

The corresponding score plot (Figure $3 \mathrm{~b}$ ) represents the experiments carried out in the space given by the first two PCs: the experiments of the initial simplex are indicated as full circles, while the other experiments performed are projected into the space given by the first two PCs calculated on the initial simplex only, and are indicated as empty squares. Experiments S7 and S11 of the initial simplex are the worst, as they are located at large negative values along $\mathrm{PC}_{1}$, while some good conditions can be identified among the other experiments of the initial simplex (e.g. experiments S6 and S4). The other experiments performed during the iterative procedure move progressively towards more positive values of $\mathrm{PC}_{1}$ (i.e. towards larger values of the calculated $\mathrm{S} / \mathrm{N}$ ). The best experimental conditions obtained correspond to experiment S60, which shows the largest positive score in $\mathrm{PC}_{1}$ (Table 4, last row). Looking at the score plot, this experiment is located at more positive scores in both the first and the second PCs than the default conditions (S1). Although an exhaustive discussion of the effects of the parameters is not within the scope of this work, a brief discussion of the effects of the most relevant parameters will now be provided. Looking at Table 4, the experimental settings corresponding to $\mathrm{S} 60$ show significant differences from the settings for $\mathrm{S} 1$ for some parameters: Mult1 shows more negative values, relating to a larger $\mathrm{S} / \mathrm{N}$ ratio, as already described for the experiments of the initial simplex; other parameters that show quite different settings

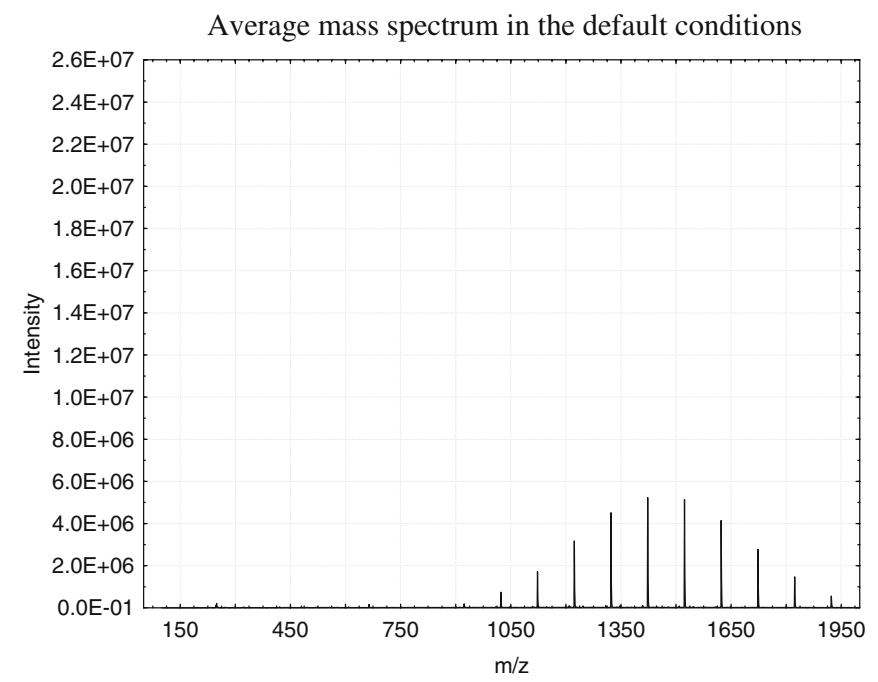

(a) compared to S1 are TLens (tube lens offset voltage) and CapTemp (the temperature of the capillary). An increase in TLens (towards the positive extreme for positive ions and towards the negative extreme for negative ions) is generally related to increased molecular fragmentation. Low values of this parameter result in low-energy collisions with the background gas, which aid in desolvating the ion. High values of this parameter result in high-energy collisions with the background gas, which can lead to collision-induced dissociation. Values ranging from 0 to 30 are usually adopted: in this case, the optimal value is about 13. For the temperature of the capillary, larger values usually enhance sample desolvation and ionization.

The default settings suggested by the manufacturer provided an average $\mathrm{S} / \mathrm{N}$ of about 164 , while the best experimental conditions identified by simplex optimization provided an average $\mathrm{S} / \mathrm{N}$ ratio of about 282 . Therefore, the tuning procedure provided an improvement in the calculated average $\mathrm{S} / \mathrm{N}$ of $71 \%$, leading to corresponding improvements in the LOD (limit of detection) and LOQ (limit of quantification) values.

Figure 4 reports the average spectra calculated over 500 scans recorded for the 1:100 diluted standard mixture when the default settings were adopted (Figure $4 \mathrm{a}$ ) and when the experimental settings corresponding to the optimal conditions identified by simplex were used (Figure 4b). A general improvement in the signal at almost all $\mathrm{m} / \mathrm{z}$ values can be clearly detected.

\section{Conclusions}

The outcome of the work presented here must not be seen merely as the application of an optimization procedure to a
Average mass spectrum for the best experimental conditions identified by simplex

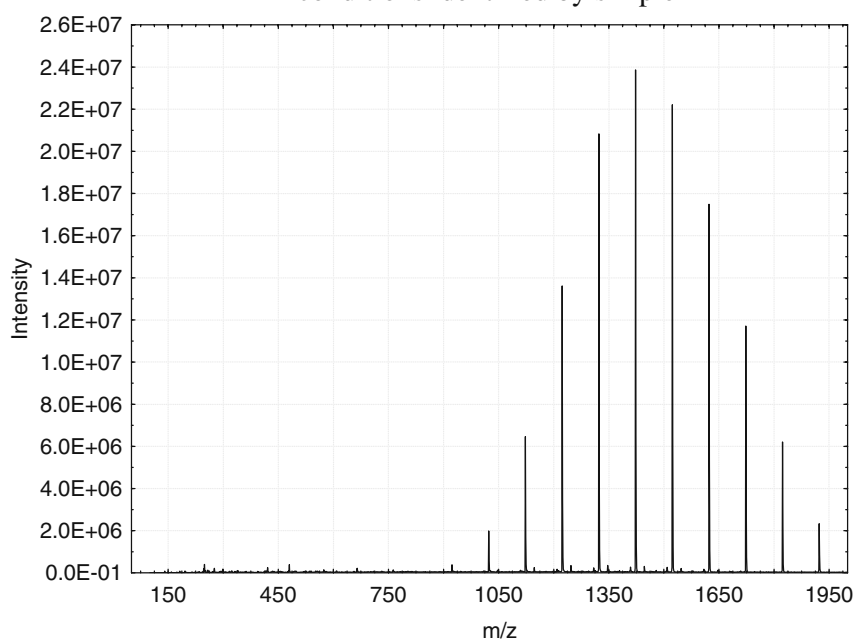

(b)

Figure 4. Comparison between the mass spectra recorded before and after simplex optimization: average mass spectrum obtained under the default conditions suggested by the manufacturer (a), and average mass spectrum recorded under the best experimental settings identified by experiment S60 of the simplex sequence (b). The average spectra were calculated over 500 subsequent scans recorded for the standard solution diluted 1:100 
particular instrumentation with no generalisation of the results. Modern instrumentation provides complex experimental responses that are quite difficult to deal with; in addition, the manufacturer often provides the final user with default conditions and best practices that must be carefully controlled and verified.

The default conditions suggested by the manufacturer in this case do not correspond to the best experimental settings; moreover, the suggested tuning procedure suffers from some drawbacks: (1) it is impossible to evaluate the interactions between the parameters to be optimised; (2) an excessively concentrated standard mixture is used, causing problems with persistency and saturation; (3) it is impossible to evaluate both signal and noise during optimization; (4) there is a lack of information about the variability of the results.

With this in mind, the work presented here could provide a modus operandi for accomplishing reliable optimization of the experimental parameters when complex instrumentation must be used. The procedure should therefore involve:

1. The identification of a proper target function

2. The identification of the experimental domain and the variations applied to each parameter to be optimised

3. The evaluation of the experimental error and a check for potential memory effects

4. The final optimization of the target function by applying the simplex methodology

The simplex optimization outlined here could also be applied to identify the best conditions for performing MSMS ion dissociation experiments. Work is in progress at our laboratories to implement the overall procedure as a completely automatic tool.

\section{References}

1. Thermoquest-Finnigan: LCQDuo-Getting Started Guide, Revision A. Thermoquest-Finnigan: San Jose (1999)

2. Applied Biosystems/MSD Analytical Technologies: Analyst SoftwareGetting Started Guide. Applied Biosystems/MSD Analytical Technologies: Carlsbad (2008)

3. Waters Corp.: Millennium32 ZQ Mass Detector Software-Getting Started Guide. Waters Corp.: Milford (2001)

4. Rogatsky, E., Stein, D.: Automated Optimization of mass spectrometric parameters in tune mode for impure analytes in dirty samples using gradient high-performance liquid chromatography. Rapid Commun. Mass Spectrom. 19, 3759-3760 (2005)

5. Carlson, R.: Design and optimization in Organic Synthesis. Elsevier, Amsterdam (1992)

6. Marengo, E., Robotti, E., Gosetti, F., Zerbinati, O., Gennaro, M.C.: Evaluation of signal and noise and identification of a suitable target function in the tuning of an ESI ion trap mass spectrometer by multivariate pattern recognition tools. J. Am. Soc. Mass Spectrom. 20(10), 1859-1867 (2009)

7. Marengo, E., Gennaro, M.C., Gianotti, V.: A simplex-optimized chromatographic separation of fourteen cosmetic preservatives: analysis of commercial products. J. Chromatogr. Sci. 39(8), 339-344 (2001)
8. Nelder, J.A., Mead, R.: A simplex algorithm for function minimization. Comput. J. 7, 308-313 (1965)

9. Carlson, R., Hansson, L., Lundstedt, T.: Optimization in organic synthesis. Strategies when the desired reaction is accompanied by parasitic side reactions. Acta Chem. Scand. B 40(6), 444-452 (1986)

10. Vanderginste, B.G.M., Massart, D.L., Buydens, L.M.C., De Jong, S., Lewi, P.J., Smeyers-Verbeke, J.: Handbook of Chemometrics and Qualimetrics: Part A. Elsevier, Amsterdam (1998)

11. Spendley, W., Hext, G.R., Himsworth, F.R.: Sequential application of simplex designs in optimization and evolutionary operation. Technometrics 4(4), 441-461 (1962)

12. Evans, C.S., Startin, J.R., Goodall, D.M., Keely, B.J.: Optimization of ion trap parameters for the quantification of chlormequat by liquid chromatography/mass spectrometry and the application in the analysis of pear extracts. Rapid Commun. Mass Spectrom. 14(2), 112-117 (2000)

13. Goodwin, L., Startin, J.R., Goodall, D.M., Keely, B.J.: Tandem mass spectrometric analysis of glyphosate, glufosinate, aminomethylphosphonic acid and methylphosphinicopropionic acid. Rapid Commun. Mass Spectrom. 17(9), 963-969 (2003)

14. Wickens, J.R., Sleeman, R., Keely, B.J.: Atmospheric pressure ionization mass spectrometric fragmentation pathways of noscapine and papaverine revealed by multistage mass spectrometry and in-source deuterium labelling. Rapid Commun. Mass Spectrom. 20(3), 473-480 (2006)

15. Marengo, E., Aceto, M., Robotti, E., Oddone, M., Bobba, M.: Optimization of sensitivity in the multi-elemental determination of 83 isotopes by ICP-MS as a function of 21 instrumental operative conditions by modified simplex, principal component analysis and partial least squares. Talanta 76(5), 1224-1232 (2008)

16. Mas, J.L., Garcia-Leon, M., Bolivar, J.P.: Tc-99 atom counting by quadrupole ICP-MS. Optimization of the instrumental response. Nucl. Instrum. Meth. A 484(1-3), 660-667 (2002)

17. Lobinski, R., Vanborm, W., Broekaert, J.A.C., Tschopel, P., Tolg, G.: Optimization of slurry nebulisation inductively-coupled plasma atomic emission spectrometry for the analysis of $\mathrm{ZrO}_{2}$ powder. Fresenius J. Anal. Chem. 342(7), 563-568 (1992)

18. Koch, I., Harrington, C.F., Reimer, K.J., Cullen, W.R.: Simplex optimization of conditions for the determination of antimony in environmental samples by using electrothermal atomic absorption spectrometry. Talanta 44(5), 771-780 (1997)

19. Ramalhosa, E., Segade, S.R., Pereira, E., Vale, C., Duarte, A.: Simple methodology for methylmercury and inorganic mercury determinations by high-performance liquid chromatography-cold vapour atomic fluorescence spectrometry. Anal. Chim. Acta 448(1-2), 135-143 (2001)

20. Cordero, T.S., Alonso, E.I.V., Rudner, P.C., de Torres, A.G., Pavon, J.M. C.: Computer-assisted SIMPLEX optimization of an on-line preconcentration system for determination of nickel in sea-water by electrothermal atomic absorption spectrometry. J. Anal. At. Spectrom. 14(7), 1033-1037 (1999)

21. March, R.E.: An introduction to quadrupole ion trap mass spectrometry. J. Mass Spectrom. 32, 351-369 (1997)

22. Reemtsma, T.: The use of liquid chromatography-atmospheric pressure ionization-mass spectrometry in water analysis, part I: achievements. Trends Anal. Chem. 20, 500-517 (2001)

23. Rosenberg, E.: The potential of organic (electrospray and atmospheric pressure chemical ionization) mass spectrometric techniques coupled to liquid-phase separation for speciation analysis. J. Chromatogr. A 1000, 841-889 (2003)

24. Wong, P.S.H., Cooks, R.G.: Ion trap mass spectrometry. Curr. Sep. 16, 85-92 (1997)

25. Massart, D.L., Vanderginste, B.G.M., Deming, S.M., Michotte, Y., Kaufman, L.: Chemometrics: A Textbook. Elsevier, Amsterdam (1988)

26. Moini, M.: Ultramark-1621 as a calibration reference compound for mass spectrometry. 2. Positive-ion and negative-ion electrospray ionization. Rapid Commun. Mass Spectrom. 8, 711-714 (1994)

27. Moini, M., Jones, B.L., Rogers, R.M., Jiang, L.: Sodium trifluoroacetate as a tune/calibration compound for positive and negative-ion electrospray ionization mass spectrometry in the mass range of 100-4000 Da. J. Am. Soc. Mass Spectrom. 9, 977-980 (1998) 